\title{
Downregulation of CD99 and upregulation of human leukocyte antigen class II promote tumor aggravation and poor survival in patients with osteosarcomas
}

\author{
This article was published in the following Dove Press journal: \\ OncoTargets and Therapy \\ 24 March 2014 \\ Number of times this article has been viewed
}

\section{Quan Zhou* \\ Jin $X u^{*}$ \\ Jiali Zhao \\ Shaoxian Zhang \\ Wei Pan}

Department of Orthopaedics, the second Hospital of Huai'an city Affiliated to Xuzhou Medical College and the second Hospital of Huai'an city, Huai'an, People's Republic of China

*These authors contributed equally to this work
Correspondence: Wei Pan Department of Orthopaedics,

The second Hospital of Huai'an city, No.62, Huaihai South Road, Huai'an City, 223002, Jiangsu Province, People's Republic of China

Tel +8651783802973

Fax +8651783947246

Email wuque197928@163.com
Background: CD99 is involved in the intracellular transport of human leukocyte antigen class II (HLA-II) protein. The aim of this study was to clarify the clinical value of CD99 and HLA-II expression in primary osteosarcoma.

Methods: One hundred and thirty pairs of osteosarcoma and matched noncancerous bone tissues were evaluated by immunohistochemistry for CD99 and HLA-II expression.

Results: Compared with the noncancerous bone tissues, the expression levels of CD99 (tumor versus normal: $2.96 \pm 0.09$ versus $5.89 \pm 1.26, P<0.001$ ) and HLA-II (tumor versus normal: $5.01 \pm 1.39$ versus $1.92 \pm 0.06, P<0.001)$ proteins were respectively downregulated and upregulated in osteosarcoma tissues. CD99 and HLA-II were highly expressed in 49/130 (37.69\%) and $107 / 130(82.31 \%)$ of osteosarcoma tissues, respectively. In addition, the osteosarcoma patients with downregulation of CD99 and upregulation of HLA-II more frequently showed the presence of metastasis and recurrence and poor response to chemotherapy. Moreover, there was a negative correlation between CD99 and HLA-II expression in osteosarcoma tissues ( $r=-0.69, P=0.01)$. The patients with low CD99 expression correlated with poor prognosis of osteosarcoma, as opposed to HLA-II. Patients with CD99-low/HLA-II-high expression had the lowest overall and disease-free survival rates, and conjoined expression of CD99/HLA-II was an independent prognostic indicator of osteosarcoma.

Conclusion: These findings suggest for the first time that CD99 downregulation or HLA-II upregulation may be an important feature of human osteosarcoma. The combined detection of CD99/HLA-II coexpression may present a predictive and prognostic indicator in osteosarcoma.

Keywords: CD99, HLA-II, osteosarcoma, immunohistochemistry, prognosis

\section{Introduction}

Osteosarcoma, as one of the most common primary malignant bone tumors in adolescents and young adults, is characterized by highly malignant and invasive growth. ${ }^{1}$ It exhibits a peak in manifestation during the second and third decades. Although the traditional treatment strategies, including wide tumor resection, neoadjuvant chemotherapy, and amputation, improve the clinical outcome of patients with osteosarcomas, pulmonary metastasis occurs in approximately $40 \%$ of patients and remains the major cause of mortality. ${ }^{2}$ The 5 -year survival rates are still less than $20 \%$. $^{3}$ In recent years, several clinicopathological features have been used as prognostic factors, albeit with discrepancies. Historically, the presence or absence 
of metastatic disease at diagnosis has been the most important prognostic factor. The response to chemotherapy is also an important and generally accepted prognostic factor; however, it is only possible after preoperative therapy and confined to different postoperative therapeutic regimens. ${ }^{4}$ Initial tumor size together with the response to chemotherapy also constitute the basis for risk-adapted postoperative chemotherapy, but drug resistance still remains a major problem. ${ }^{5}$ Given the lack of reliable prognostic factors for patients with osteosarcomas, the identification of novel and efficient diagnostic and prognostic markers is especially important for the prediction of tumor response to chemotherapy, the development of individually adapted multimodality treatments, and the improvement of clinical outcome of patients.

CD99 is a $32 \mathrm{kDa}$, transmembrane protein encoded by the $M I C 2$ gene, located on the pseudoautosomal region at the tips of the short arms of the $\mathrm{X}$ and $\mathrm{Y}$ chromosomes. ${ }^{6}$ As a sialomucin-type glycoprotein, CD99 is ubiquitously expressed, albeit to varying degrees, by different cell types. Functionally, it is implicated in various cellular events such as cell adhesion and migration, apoptosis, cell differentiation, and regulation of protein trafficking. ${ }^{7}$ Biologically, CD99 protein is expressed in a wide variety of normal tissues, particularly in the mature plasma cells of reactive lymph nodes, cortical thymocytes, pancreatic islet cells, granulosa cells of the ovaries, and Sertoli cells of the testes. ${ }^{8}$ In addition, the reduced expression of CD99 has been observed and has been suggested to act as a tumor suppressor of various kinds of malignancies, such as Ewing's sarcoma/ primitive neuroectodermal tumors, pancreatic endocrine tumors, anaplastic large cell lymphoma, lymphoblastic lymphoma/leukemia, transitional cell carcinoma of urinary bladder, gastric adenocarcinoma, rhabdomyosarcoma, breast cancer, ovarian cancer, and pleomorphic carcinomas of the lung. ${ }^{9-12}$ In particular, CD99 functions as an antioncogenic factor in osteosarcoma. Manara et $\mathrm{al}^{13}$ in 2006 found that the forced expression of CD99 in two osteosarcoma cell lines significantly reduced resistance to anoikis, inhibited growth in anchorage independence as well as cell migration, and led to abrogation of tumorigenic and metastatic ability. Mayordomo et $\mathrm{al}^{14}$ in 2010 showed the expression of CD99 in osteosarcoma by immunohistochemistry. In 2013, Zucchini et $\mathrm{al}^{15}$ indicated that CD99 could suppress osteosarcoma cell migration through inhibition of Rho-associated, coiled-coilcontaining protein kinase 2 activity. However, the clinical and pathological relevance of CD99 in osteosarcoma has been rarely studied.
Immune surveillance plays a crucial role in curbing the process of tumorigenesis, which depends on the genetic instability of tumors and interactions with their immunological environment. Human leukocyte antigens (HLA) are involved in mounting immune responses against tumor antigens by recruiting cytotoxic T lymphocytes, which leads to tumor evasion and destruction. ${ }^{16}$ HLA class II (HLA-II), in particular, is implicated in antigen presentation to the immune system. ${ }^{17}$ Considering its key functions, it has been already demonstrated that an allele at HLA-II locus as well as regulated HLA-II expression are important for the control of the immune response and are associated to diseases. Recent studies have demonstrated that the alterations of HLA-II play key roles in the development and metastatic progression of various human cancers, including breast cancer, malignant melanoma, colorectal, and gastric cancer. ${ }^{18-20}$ Moreover, Baccar et $\mathrm{al}^{21}$ and Yoon et $\mathrm{al}^{22}$ indicated that CD99 may be involved in the intracellular transport of surface molecules, such as the $\mathrm{T}$ cell receptor complex and HLA-II proteins. Considering the interaction between CD99 and HLA-II proteins and the involvement of CD99 in osteosarcoma, we propose the hypothesis that HLA-II might also play a role in tumor progression of osteosarcoma. In the present work, our aim is to clarify the clinical value of CD99 and HLA-II expression in primary osteosarcoma.

\section{Materials and methods Patients and tissue samples}

This study was approved by the Research Ethics Committee of Huai'an Hospital Affiliated to Xuzhou Medical College, People's Republic of China (No HA20130068). Written informed consent was obtained from all of the patients. All specimens were handled and made anonymous according to the ethical and legal standards.

One hundred and thirty tumor tissues and self-paired noncancerous bone tissues from 130 osteosarcoma patients were retrospectively selected from the surgical pathology records of the Huai'an Hospital Affiliated to Xuzhou Medical College from 2000 to 2008. Patients underwent X-ray imaging, computed tomography (CT), magnetic resonance imaging, and bone scintigraphy for the diagnosis. Specimens of all the tumors were obtained from surgery and confirmed pathologically. All of the tissues analyzed were obtained after treatment with neoadjuvant chemotherapy, and all patients received the uniform preoperative, multiagent chemotherapy following the initial biopsy. The cytotoxic drugs used as preoperative chemotherapy were cis-diamminedichloroplatinum, adriamycin, vincristine, ifosfamide, and high-dose methotrexate. 
Resected specimens were analyzed histologically for response to chemotherapy according to the pathological reports released previously, and corresponding clinical information was obtained from medical records. ${ }^{23}$ The clinical and pathologic parameters were obtained from the pathological reports and are presented in Table 1.

Follow-up was performed for all 130 patients from 3-102 months (median, 48 months) and ended June 30, 2013. During this period, patients were monitored with CT performed every 3 months during the first 3 years after chemotherapy, every 4 months during years 4 and 5 , and every 6 months thereafter. The development of local recurrence and distant metastases was detected by CT scans or magnetic resonance imaging. All cases were independently reviewed by two pathologists, and discrepancies resolved by consensus review. Of 130 patients with osteosarcomas, 88 (67.69\%) patients were alive and $42(32.31 \%)$ patients had died of the disease. Overall survival time was calculated from the date of the initial surgical operation to death. Disease-free survival was calculated from the date of the initial surgical operation to the date of a second cancer, tumor recurrence, distant metastases, or death from any cause.

\section{Immunohistochemical analysis}

CD99 and HLA-II expression was detected by immunohistochemistry assay. Utilizing the avidin-biotin-peroxidase complex method, formalin-fixed, paraffin-embedded, $3 \mu \mathrm{m}$ thick tissue sections were processed by brief proteolytic digestion and peroxidase blocking. Sections were then incubated with primary antibodies against CD99 (monoclonal mouse anti-human CD99, 12E7, dakocytomation M3601; dilution of 1:50) and HLA-II (monoclonal mouse anti-human HLA-II, dakocytomation M0775; dilution of 1:50) (DakoCytomation, Glostrup, Denmark) overnight at $4^{\circ} \mathrm{C}$. After washing the sections, peroxidase-labeled polymer and substrate-chromogen (Enzo Life Sciences, Inc., Farmingdale, NY, USA) solutions were applied to visualize stained proteins of interest. CD99 and HLA-II overexpression, confirmed by Western blot, was used as positive controls, and negative controls were carried out by omitting the primary antibodies.

Table I Associations of CD99 and HLA-II expressions with the clinicopathological features of primary osteosarcoma

\begin{tabular}{|c|c|c|c|c|c|c|c|}
\hline \multirow{2}{*}{$\begin{array}{l}\text { Clinicopathological } \\
\text { features }\end{array}$} & \multirow{2}{*}{$\begin{array}{l}\text { Number } \\
\text { of cases (\%) }\end{array}$} & \multicolumn{3}{|l|}{ CD99 } & \multicolumn{3}{|l|}{ HLA-II } \\
\hline & & High (\%) & Low (\%) & $P$ & High (\%) & Low (\%) & $P$ \\
\hline \multicolumn{8}{|l|}{ Age (years) } \\
\hline$<20$ & $58(44.62)$ & $19(32.76)$ & $39(67.24)$ & \multirow[t]{2}{*}{ NS } & $50(82.21)$ & 8 (I7.79) & \multirow[t]{2}{*}{ NS } \\
\hline$\geq 20$ & $72(55.38)$ & $30(4 \mid .67)$ & $42(58.33)$ & & 67 (93.06) & $5(6.84)$ & \\
\hline \multicolumn{8}{|l|}{ Sex } \\
\hline Male & 92 (70.77) & $36(39.13)$ & $56(60.87)$ & \multirow[t]{2}{*}{ NS } & 77 (83.70) & $15(16.30)$ & \multirow[t]{2}{*}{ NS } \\
\hline Female & $38(29.23)$ & I3 (34.2I) & $25(65.79)$ & & $30(73.33)$ & $8(26.67)$ & \\
\hline \multicolumn{8}{|l|}{ Tumor size } \\
\hline$\leq 5 \mathrm{~cm}$ & $60(46.15)$ & $22(36.67)$ & $38(63.33)$ & \multirow[t]{2}{*}{ NS } & $51(85.00)$ & $9(15.00)$ & \multirow[t]{2}{*}{ NS } \\
\hline$>5 \mathrm{~cm}$ & $70(53.85)$ & $27(38.57)$ & $43(61.43)$ & & $56(80.00)$ & $14(20.00)$ & \\
\hline \multicolumn{8}{|l|}{ Tumor location } \\
\hline Femur & $60(46.15)$ & $18(30.00)$ & $42(70.00)$ & \multirow[t]{4}{*}{ NS } & 44 (73.33) & $16(26.67)$ & \multirow[t]{4}{*}{ NS } \\
\hline Tibia & 40 (30.77) & $14(35.00)$ & $26(65.00)$ & & $36(90.00)$ & $4(10.00)$ & \\
\hline Humerus & $24(18.45)$ & 14 (58.33) & $10(41.67)$ & & $24(100.00)$ & $0(0)$ & \\
\hline Fibula & $6(4.62)$ & $3(50.00)$ & $3(50.00)$ & & $3(50.00)$ & $3(50.00)$ & \\
\hline \multicolumn{8}{|l|}{ Metastasis } \\
\hline No & $73(56.15)$ & 40 (54.79) & $33(45.21)$ & \multirow[t]{2}{*}{0.009} & $53(72.60)$ & $20(27.40)$ & \multirow[t]{2}{*}{0.012} \\
\hline Yes & $57(43.85)$ & $9(15.79)$ & $48(84.21)$ & & 54 (94.74) & $3(5.26)$ & \\
\hline \multicolumn{8}{|l|}{ Recurrence } \\
\hline No & $76(58.46)$ & $44(57.89)$ & $32(42.11)$ & \multirow[t]{2}{*}{0.009} & 55 (72.37) & $21(27.63)$ & \multirow[t]{2}{*}{0.010} \\
\hline Yes & $54(4 I .54)$ & $5(9.26)$ & $49(80.74)$ & & $52(96.30)$ & $2(3.70)$ & \\
\hline \multicolumn{8}{|l|}{ Histological subtype } \\
\hline Osteoblastic & $70(53.85)$ & $22(31.43)$ & $48(68.57)$ & \multirow[t]{5}{*}{ NS } & $60(85.7 \mathrm{I})$ & $10(14.29)$ & \multirow[t]{5}{*}{ NS } \\
\hline Chondroblastic & $28(21.54)$ & $14(50.00)$ & $14(50.00)$ & & $20(71.43)$ & $8(28.57)$ & \\
\hline Fibroblastic & $21(16.15)$ & $9(42.86)$ & $12(57.14)$ & & I8 (85.7I) & $3(14.29)$ & \\
\hline Mixed type & $6(4.62)$ & $2(33.33)$ & $4(66.67)$ & & $5(83.33)$ & I (16.67) & \\
\hline Small type & $5(3.85)$ & $2(40.00)$ & $3(60.00)$ & & $4(80.00)$ & I (20.00) & \\
\hline \multicolumn{8}{|c|}{ Response to chemotherapy } \\
\hline Good & $75(53.85)$ & $34(46.67)$ & $4 I(53.33)$ & \multirow[t]{2}{*}{0.016} & $66(88.00)$ & $9(12.00)$ & \multirow[t]{2}{*}{0.022} \\
\hline Poor & $55(46.15)$ & $15(27.27)$ & $40(72.73)$ & & $41(74.54)$ & $14(15.46)$ & \\
\hline
\end{tabular}

Abbreviations: HLA-II, human leukocyte antigen class II; NS, no statistically significant difference. 
Two experienced pathologists, who were blinded to the clinicopathological parameters and clinical outcomes of the patients, independently scored the immunostaining according to the evaluation methods of previous studies. ${ }^{24-26}$ The scores were compared, and sections yielding discrepant scores were subjected to reexamination by both pathologists working together to achieve a consensus score. The number of positive-staining cells showing immunoreactivity on the cytoplasm (for CD99) and membrane/cytoplasm (for HLA-II) in ten representative microscopic fields were counted, and the percentage of positive cells were calculated. The percentage scoring of immunoreactive tumor cells was as follows: $0(0 \%), 1(1 \%-10 \%), 2(11 \%-50 \%)$, and $3(>50 \%)$. The staining intensity was visually scored and stratified as follows: 0 (negative), 1 (weak), 2 (moderate), and 3 (strong). An immunoreactive score (IRS) was obtained for each case by multiplying the percentage and the intensity score. Therefore, tumors with a product score exceeding 3 (median of total scores for CD99) and 5 (median of total scores for HLA-II) were respectively deemed to be high expressions of CD99 and HLA-II; all other scores were considered to be low expressions.

\section{Statistical analyses}

The software of SPSS version 13.0 for Windows (IBM Corporation, Armonk, NY, USA) was used. Statistical analyses were performed using Fisher's exact test for any $2 \times 2$ tables, Pearson chi-square test for tables not $2 \times 2$, chisquare trend test for ordinal datum, and Kaplan-Meier and Cox regression methods for the question of survival analysis. Spearman rank correlation was used to analyze the correlation between the expression of CD99 and HLA-II. Differences were considered statistically significant when $P<0.05$.

\section{Results \\ CD99 downregulation and HLA-II upregulation \\ in human osteosarcoma tissues}

Expression patterns and subcellular localizations of CD99 and HLA-II proteins in 130 patients with osteosarcomas were examined immunohistochemically. Staining results are shown in Figure 1. CD99 protein was moderately or weakly expressed in the cytoplasm and cell membrane of tumor cells in osteosarcoma tissue (Figure 1A), whereas HLA-II immunostaining was intense and predominantly located in the cytoplasm and cell membrane of osteosarcoma tissue (Figure 1B). In addition, compared with noncancerous bone tissues, the expression levels of CD99 (IRS for tumor versus normal:

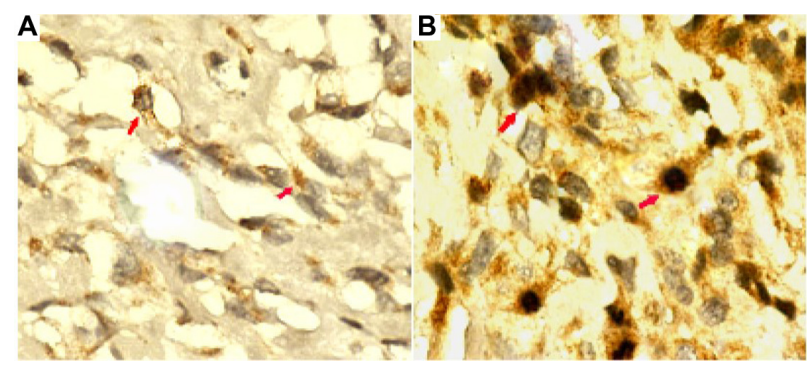

Figure I Immunohistochemical expression for CD99 and HLA-II in primary osteosarcoma tissues $(\times 400)$.

Notes: CD99 was weakly expressed in the cytoplasm and cell membrane of osteosarcoma cells (A). HLA-II was strongly expressed in the cytoplasm and cell membrane of osteosarcoma cells (B). The red arrows indicate the positive area in the osteosarcoma tissues.

Abbreviation: HLA-II, human leukocyte antigen class II.

$2.96 \pm 0.09$ versus $5.89 \pm 1.26, P<0.001)$ and HLA-II (IRS for tumor versus normal: $5.01 \pm 1.39$ versus $1.92 \pm 0.06, P<0.001$ ) proteins were respectively downregulated and upregulated in osteosarcoma tissues. Moreover, CD99 and HLA-II expression were highly expressed in 49/130 (37.69\%) and 107/130 $(82.31 \%)$ of osteosarcoma tissues, respectively. There was a negative correlation between CD99 and HLA-II expression in osteosarcoma tissues $(r=-0.69, P=0.01)$.

\section{Association of the CD99 and HLA-II expressions with the clinicopathological characteristics of human osteosarcoma}

The association of CD99 and HLA-II expression with the clinicopathological features of osteosarcoma patients is shown in Table 1 . The analysis revealed that the patients with downregulation of CD99 and upregulation of HLA-II more frequently showed the positive status of metastasis $(P=0.009$ and $P=0.012$, respectively), recurrence ( $P=0.009$ and $P=0.010$, respectively), and poor response to chemotherapy $(P=0.016$ and $P=0.022$, respectively). There was no significant association with age, sex, tumor size, tumor location, or histological subtype (Table 1).

\section{Prognostic implications of CD99 and HLA-II expression in osteosarcoma}

The prognostic value of CD99 and HLA-II expression in human osteosarcoma was further investigated by KaplanMeier analysis and the log-rank test. As shown in Figure 2, osteosarcoma tissues with low CD99 expression and high HLA-II expression were correlated with both shorter overall survival $(P<0.001)$ (Figure $2 \mathrm{~A}$ and $\mathrm{C})$ and shorter diseasefree survival $(P<0.001)$ (Figure $2 \mathrm{~B}$ and $\mathrm{D})$. According to the conjoined expressions of CD99/HLA-II, the subjects were categorized into four groups: CD99-low/HLA-II-low $(n=4)$, 

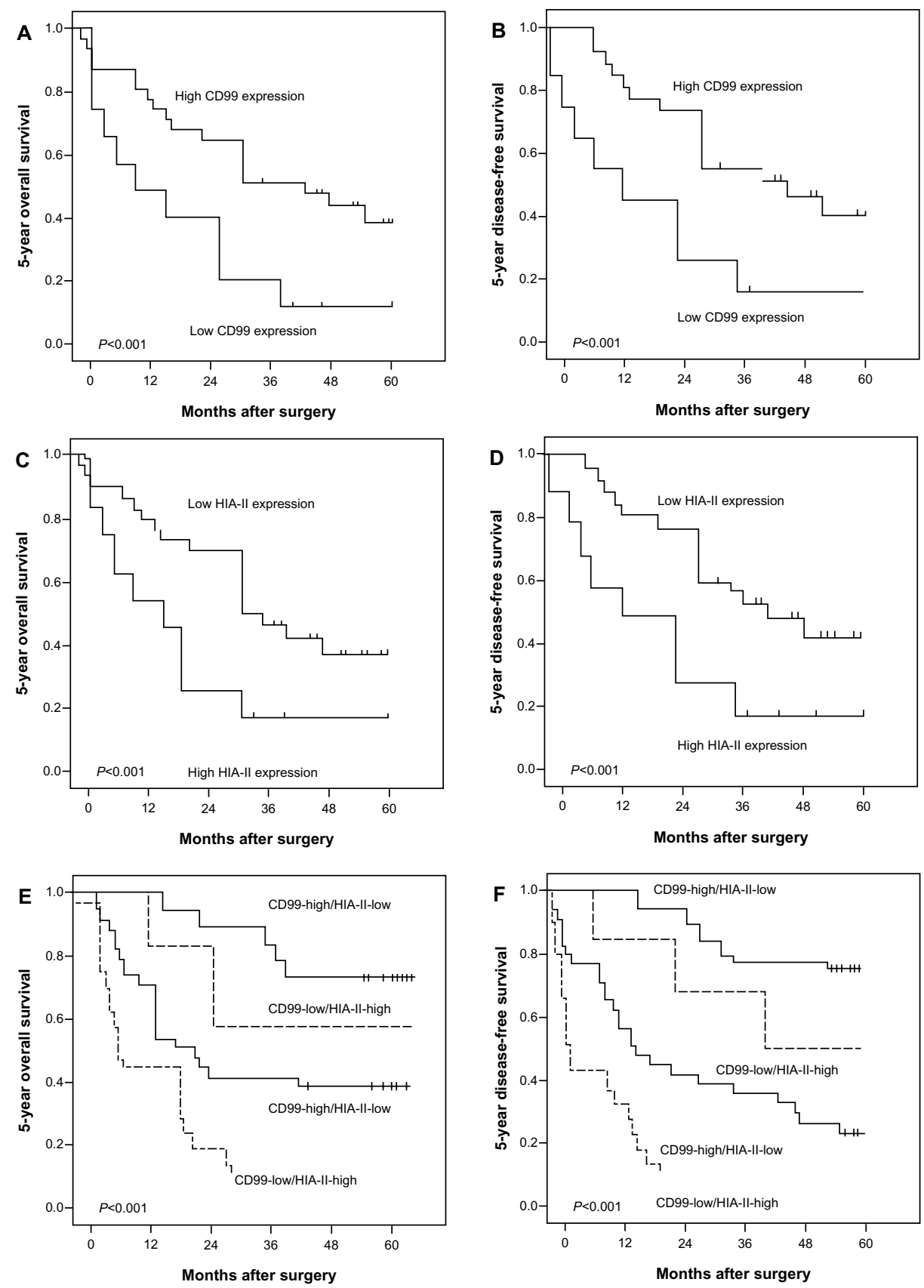

Figure 2 Kaplan-Meier survival curves of patients with primary osteosarcoma according to the expressions of CD99 (A and $\mathbf{B})$ and HLA-II (C and $\mathbf{D})$, and the coexpression of CD99/HLA-II (E and F).

Notes: The osteosarcoma patients with low CD99 expression and high HLA-II expression were correlated with both shorter overall survival (both $P<0.00 \mathrm{I})$ and shorter disease-free survival (both $P<0.00 \mathrm{I}$ ). According to the conjoined expressions of CD99/HLA-II, the subjects were categorized into four groups: CD99-low/HLA-II-low ( $=4$ ), CD99-low/HLA-II-high ( $\mathrm{n=77}$ ), CD99-high/HLA-II-low ( $\mathrm{n=19}$ ), and CD99-high/HLA-II-high $(\mathrm{n}=30)$. The association between coexpression of CD99/HLA-II and the survival rates were also tested by the method of Kaplan-Meier. The results by pairwise comparisons showed that a statistically significant difference of survival rates existed between CD99-low/HLA-II-high cases and any of other three groups $(P<0.00 I)$. In all four groups, CD99-low/HLA-II-high subjects had the poorest prognosis.

Abbreviation: HLA-II, human leukocyte antigen class II.

CD99-low/HLA-II-high (n=77), CD99-high/HLA-II-low ( $\mathrm{n}=19$ ), and CD99-high/HLA-II-high ( $\mathrm{n}=30$ ). The association between coexpression of CD99/HLA-II and the survival rates were also tested by the method of Kaplan-Meier. The results by pairwise comparisons showed that a statistically significant difference of survival rates existed between CD99-low/
HLA-II-high cases and any of the other three groups $(P<0.001)$ (Figure 2E and F). Of all four groups, CD99-low/ HLA-II-high subjects had the poorest prognosis.

In addition, univariate analysis showed that the positive recurrence and metastasis, the low expression of CD99, the high expression of HLA-II protein, the combined CD99-low/ 
HLA-II-high expression, and the poor response to chemotherapy were all significantly correlated with poor overall survival $(P=0.006, P<0.001, P<0.001, P<0.001$, and $P<0.001$, respectively) (Table 2$)$ and disease-free survival $(P=0.01$, $P<0.001, P<0.001, P<0.001$, and $P<0.001$, respectively) (Table 2) of osteosarcoma patients.

Furthermore, multivariate analysis as shown in Table 3 demonstrated that the positive recurrence (both $P=0.022$ ) and metastasis ( $P=0.009$ and $P=0.013$, respectively), the low expression of CD99 (both $P=0.010$ ), the high expression of HLA-II protein ( $P=0.008$ and $P=0.012$, respectively), the combined CD99-low/HLA-II-high expression (both $P=0.002)$ and the poor response to chemotherapy $(P=0.011$ and $P=0.016$, respectively) were all independent prognostic factors for overall survival and disease-free survival.

\section{Discussion}

The prognosis of osteosarcoma patients with distant metastasis and local recurrence is still poor, even with extensive chemotherapy. Although there are a large number of prognostic

Table 2 Univariate analysis of prognostic parameters in patients with osteosarcomas by Cox regression analysis

\begin{tabular}{|c|c|c|c|c|}
\hline \multirow[t]{2}{*}{ Variables } & \multicolumn{2}{|c|}{ Overall survival } & \multicolumn{2}{|c|}{$\begin{array}{l}\text { Disease-free } \\
\text { survival }\end{array}$} \\
\hline & $P$-value & $\begin{array}{l}\text { Relative } \\
\text { risk }\end{array}$ & $P$-value & $\begin{array}{l}\text { Relative } \\
\text { risk }\end{array}$ \\
\hline \multicolumn{5}{|l|}{ Age at diagnosis (years) } \\
\hline$<20$ versus $\geq 20$ & 0.125 & 0.691 & 0.118 & 0.651 \\
\hline \multicolumn{5}{|l|}{ Sex } \\
\hline Male versus female & 0.112 & 0.916 & 0.110 & 0.846 \\
\hline \multicolumn{5}{|l|}{ Tumor size $(\mathrm{cm})$} \\
\hline$<5.0$ versus $\geq 5.0$ & 0.312 & 0.568 & 0.310 & 0.521 \\
\hline \multicolumn{5}{|l|}{ Tumor location } \\
\hline $\begin{array}{l}\text { Femur versus tibia } \\
\text { and other }\end{array}$ & 0.200 & 0.899 & 0.213 & 0.887 \\
\hline \multicolumn{5}{|l|}{ Histological subtype } \\
\hline $\begin{array}{l}\text { Osteoblastic versus } \\
\text { other }\end{array}$ & 0.116 & 0.925 & 0.119 & 0.921 \\
\hline \multicolumn{5}{|l|}{ Recurrence } \\
\hline No versus yes & 0.006 & 4.732 & 0.01 & 4.098 \\
\hline \multicolumn{5}{|l|}{ Metastasis } \\
\hline No versus yes & $<0.001$ & 8.279 & $<0.001$ & 8.132 \\
\hline \multicolumn{5}{|c|}{ Response to chemotherapy } \\
\hline Good versus poor & $<0.00 \mathrm{I}$ & 8.026 & $<0.001$ & 7.969 \\
\hline \multicolumn{5}{|l|}{ CD99 expression } \\
\hline Low versus high & $<0.001$ & 7.408 & $<0.001$ & 7.064 \\
\hline \multicolumn{5}{|l|}{ HLA-II expression } \\
\hline Low versus high & $<0.001$ & 7.036 & $<0.001$ & 7.028 \\
\hline \multicolumn{5}{|c|}{ CD99/HLA-II expression } \\
\hline $\begin{array}{l}\text { CD99-low/HLA-II- } \\
\text { high versus others }\end{array}$ & $<0.001$ & 8.482 & $<0.001$ & 8.359 \\
\hline
\end{tabular}

Abbreviation: HLA-II, human leukocyte antigen class II. factors for patients with osteosarcoma, including age, sex, truncal location, metastatic lesions at initial diagnosis, response to chemotherapy, tumor size, alkaline phosphatase, recurrence, and P-glycoprotein levels, the accuracy of these factors is not satisfactory. Thus, it is a great challenge to clinicians and basic scientists to identify the molecular markers associated with the progression and prognosis of osteosarcoma. The data presented in this study show evidence of downregulation of CD99 and upregulation of HLA-II in osteosarcoma tissues, which may promote tumor aggravation and predict poor prognosis in patients with osteosarcomas. To the best of our knowledge, this is the first investigation of the clinical significance of CD99 and HLA-II in human osteosarcoma.

CD99 is expressed in most human tissues, and its expression has been observed in a wide variety of neoplasms including breast cancer, gastric cancer, lung cancer, Ewing's sarcoma/ primitive neuroectodermal tumor, osteosarcoma, and pancreatic neuroendocrine carcinoma. ${ }^{9-12}$ The membranous and cytoplasmic staining patterns have been interpreted as significantly positive expression of CD99. ${ }^{27}$ Interestingly, the expression levels are increased or decreased in various human cancers, suggesting it may have a tumor type-dependent expression pattern. For example, CD99 expression was significantly upregulated in breast cancer tissues as compared to nonmalignant breast tissue; ${ }^{9}$ the invasive squamous cell carcinoma showed significantly increased expression of CD99 when compared with normal cervix tissues, ${ }^{10}$ and the upregulation of CD99 was observed in pleomorphic carcinoma of the lung. ${ }^{11}$ In contrast, the positive rate of CD99 protein expression in transitional cell carcinoma of urinary bladder tissues was lower than in normal urinary bladder epithelia; ${ }^{12}$ CD99 expression was downregulated in pancreatic endocrine tumor and loss of CD99 expression was related to markers of worse prognosis for this tumor, including gross local invasion, metastasis to the lymph nodes or other organs, and lymphatic or blood vessel invasion. ${ }^{28}$ In the current study, several pieces of evidence suggest that CD99 might function as a candidate suppressor for osteosarcoma, which was in line with the findings of Manara et $\mathrm{al}^{13}$ and Zucchini et al. ${ }^{15}$ First, the downregulation of CD99 expression is frequently observed in the majority of osteosarcomas compared with noncancerous tissues. Second, the low CD99 expression is significantly associated with malignant tumor features. Immunohistochemical analysis of CD99 protein expression in osteosarcoma indicated that CD99 expression loss is closely correlated with the positive status of recurrence and metastasis and poor response of chemotherapy. Both the univariate and multivariate survival analyses showed that the overall survival and disease-free survival of the patients with low CD99 expression were shorter than 
Table 3 Multivariate analysis of prognostic parameters in patients with osteosarcomas by Cox regression analysis

\begin{tabular}{|c|c|c|c|c|}
\hline \multirow[t]{2}{*}{ Prognostic factors } & \multicolumn{2}{|l|}{ Overall survival } & \multicolumn{2}{|l|}{ Disease-free survival } \\
\hline & Relative risk $(95 \% \mathrm{CI})$ & $P$-value & Relative risk $(95 \% \mathrm{CI})$ & $P$-value \\
\hline Recurrence & $2.167(0.980-4.56 \mathrm{I})$ & 0.022 & $2.020(0.972-4.416)$ & 0.022 \\
\hline Metastasis & $5.020(1.051-11.109)$ & 0.009 & $5.006(1.109-11.088)$ & 0.013 \\
\hline Response to chemotherapy & $3.252(0.932-7.867)$ & 0.010 & $3.158(0.973-7.567)$ & 0.010 \\
\hline CD99 expression & $5.019(1.202-11.229)$ & 0.008 & $5.108(1.200-11.862)$ & 0.012 \\
\hline HLA-II expression & $3.128(1.001-6.929)$ & 0.011 & $3.012(1.00 \mathrm{I}-6.042)$ & 0.016 \\
\hline CD99/HLA-II expression & $6.862(1.39|-| 4.882)$ & 0.002 & $6.833(1.322-14.689)$ & 0.002 \\
\hline
\end{tabular}

Abbreviations: $\mathrm{Cl}$, confidence interval; HLA-II, human leukocyte antigen class II.

those with high expression. Lee et $\mathrm{al}^{29}$ have reported that the observed downregulation of CD99 expression in gastric adenocarcinoma cases may be due to CD99 promoter methylation and downregulation of the transcription factor sp1. However, the possible mechanism for the aberrant expression of CD99 acting on human osteosarcoma is still unclear and merits further investigation.

Since $T$ cells fail to recognize nonself antigens in the absence of HLA peptide complex, the HLA-II protein appears integral to the antigen presentation process, a crucial step in eliciting an efficient immune response. ${ }^{17}$ Accumulating studies have indicated that the alterations of HLA-II protein may be relevant to the development of various human malignancies. For example, Chaudhuri et $\mathrm{al}^{18}$ found that the genetic variants in HLA-II alleles might represent resistance factors toward breast cancer. Younger et al ${ }^{19}$ suggested that malignant prostate cancer may be targeted via the HLA-II pathway, and that class II-positive tumors could be employed for direct antigen presentation. Kübler et $\mathrm{al}^{20}$ also observed the genetic alterations of HLA-II in ovarian cancer, and Chuang et $\mathrm{a}^{30}$ indicated that HLA-II genotypes may play important roles in determining high viral load and persistent infection of human papilloma virus-18 and subsequent cervical neoplasia risk. In the present study, we found that HLA-II was overexpressed in the osteosarcoma tissues compared to noncancerous bone tissues, and higher HLA-II expression was significantly associated with the positive status of recurrence and metastasis, the poor response of chemotherapy, and shorter overall and disease-free survival. In contrast, Baccar et $\mathrm{al}^{21}$ reported better outcomes in breast cancer when HLA-II expression was increased, suggesting that the different roles of HLA-II depend on various tumor types. However, the possible mechanism for the aberrant expression of HLA-II acting on human osteosarcoma remains unclear and warrants further investigation.

Interestingly, we determined that, among four different states of CD99/HLA-II coexpression, the osteosarcoma patients with CD99-high/HLA-II-low expression had the best outcome, while those with CD99-low/HLA-II-high had the worst. Multivariate analysis revealed that CD99-low/HLA-II-high coexpression may be an independent prognostic indicator for osteosarcoma. Our results show the advantages of CD99/HLA-II coexpression over individual CD99 or HLA-II in predicting the outcome of osteosarcoma. However, the effect of chemotherapy on these markers is unknown since paired samples obtained at pretreatment and posttreatment were not available.

In conclusion, these findings suggest that CD99 downregulation or HLA-II upregulation may be an important feature of human osteosarcoma. The combined detection of CD99/ HLA-II coexpression may be of benefit in predicting prognosis in osteosarcoma patients. Our data also suggest a relationship between CD99/HLA-II coexpression and prognosis in patients with osteosarcoma. However, there is a limitation in the current study. The most favorable group (CD99-low/HLA-II-high) only comprises less than $15 \%$ of patients, which may preclude significance. The findings in this study suggest the importance of further prospective analysis of a large cohort.

\section{Acknowledgments}

This research was supported by grants from the scientific research fund of the Bureau of Public Health of Jiangsu province (No H201254) and the Huai' an Technology Support Program (social development) funded projects (No HAS2013028).

\section{Author contributions}

Wei Pan designed the study. Quan Zhou and Jin Xu performed the experiments. Quan Zhou, Jin Xu, Jiali Zhao and Shaoxian Zhang performed the data analysis. All authors took part in drafting the article or revising it critically for important intellectual content. All authors approved the final version to be published.

\section{Disclosure}

The authors report no conflicts of interest in this work.

\section{References}

1. Ottaviani G, Jaffe N. The epidemiology of osteosarcoma. Cancer Treat Res. 2009;152:3-13. 
2. Gill J, Ahluwalia MK, Geller D, Gorlick R. New targets and approaches in osteosarcoma. Pharmacol Ther. 2013;137(1):89-99.

3. Allison DC, Carney SC, Ahlmann ER, et al. A meta-analysis of osteosarcoma outcomes in the modern medical era. Sarcoma. 2012;2012: 704872.

4. Tabone MD, Kalifa C, Rodary C, Raquin M, Valteau-Couanet D, Lemerle J. Osteosarcoma recurrences in pediatric patients previously treated with intensive chemotherapy. J Clin Oncol. 1994;12(12): 2614-2620.

5. Trieb K, Kotz R. Proteins expressed in osteosarcoma and serum levels as prognostic factors. Int J Biochem Cell Biol. 2001;33(1):11-17.

6. Ambros IM, Ambros PF, Strehl S, Kovar H, Gadner H, SalzerKuntschik M. MIC2 is a specific marker for Ewing's sarcoma and peripheral primitive neuroectodermal tumors. Evidence for a common histogenesis of Ewing's sarcoma and peripheral primitive neuroectodermal tumors from MIC2 expression and specific chromosome aberration. Cancer. 1991;67(7):1886-1893.

7. Seol HJ, Chang JH, Yamamoto J, et al. Overexpression of CD99 Increases the Migration and Invasiveness of Human Malignant Glioma Cells. Genes Cancer. 2012;3(9-10):535-549.

8. Hahn JH, Kim MK, Choi EY, et al. CD99 (MIC2) regulates the LFA-1/ ICAM-1-mediated adhesion of lymphocytes, and its gene encodes both positive and negative regulators of cellular adhesion. J Immunol. 1997;159(5):2250-2258.

9. Byun HJ, Hong IK, Kim E, et al. A splice variant of CD99 increases motility and MMP-9 expression of human breast cancer cells through the AKT-, ERK-, and JNK-dependent AP-1 activation signaling pathways. J Biol Chem. 2006;281(46):34833-34847.

10. Zhou JH, Ye F, Chen HZ, Zhou CY, Lu WG, Xie X. Altered expression of cellular membrane molecules of HLA-DR, HLA-G and CD99 in cervical intraepithelial neoplasias and invasive squamous cell carcinoma. Life Sci. 2006;78(22):2643-2649.

11. Edlund K, Lindskog C, Saito A, et al. CD99 is a novel prognostic stromal marker in non-small cell lung cancer. Int $J$ Cancer. 2012;131(10): 2264-2273.

12. Jung KC, Park WS, Bae YM, et al. Immunoreactivity of CD99 in stomach cancer. J Korean Med Sci. 2002;17(4):483-489.

13. Manara MC, Bernard G, Lollini PL, et al. CD99 acts as an oncosuppressor in osteosarcoma. Mol Biol Cell. 2006;17(4):1910-1921.

14. Mayordomo E, Machado I, Giner F, et al. A tissue microarray study of osteosarcoma: histopathologic and immunohistochemical validation of xenotransplanted tumors as preclinical models. Appl Immunohistochem Mol Morphol. 2010;18(5):453-461.

15. Zucchini C, Manara MC, Pinca RS, et al. CD99 suppresses osteosarcoma cell migration through inhibition of ROCK2 activity. Oncogene. Epub May 6, 2013.

16. Igney FH, Krammer PH. Death and anti-death: tumour resistance to apoptosis. Nat Rev Cancer. 2002;2(4):277-288.
17. Baccar Harrath A, Yacoubi Loueslati B, Troudi W, et al. HLA class II polymorphism: protective or risk factors to breast cancer in Tunisia? Pathol Oncol Res. 2006;12(2):79-81.

18. Chaudhuri S, Cariappa A, Tang M, et al. Genetic susceptibility to breast cancer: HLA DQB*03032 and HLA DRB1*11 may represent protective alleles. Proc Natl Acad Sci U SA. 2000;97(21):11451-11454.

19. Younger AR, Amria S, Jeffrey WA, et al. HLA class II antigen presentation by prostate cancer cells. Prostate Cancer Prostatic Dis. 2008;11(4): 334-341.

20. Kübler K, Arndt PF, Wardelmann E, et al. Genetic alterations of HLAclass II in ovarian cancer. Int J Cancer. 2008;123(6):1350-1356.

21. Baccar A, Ferchichi I, Troudi W, et al. CD99 and HLA-II immunostaining in breast cancer tissue and their correlation with lymph node metastasis. Dis Markers. 2013;34(5):363-371.

22. Yoon SS, Kim HJ, Chung DH, Kim TJ. CD99 costimulation up-regulates $\mathrm{T}$ cell receptor-mediated activation of JNK and AP-1. Mol Cells. 2004;18(2):186-191.

23. Bielack SS, Kempf-Bielack B, Delling G, et al. Prognostic factors in high-grade osteosarcoma of the extremities or trunk: an analysis of 1,702 patients treated on neoadjuvant cooperative osteosarcoma study group protocols. J Clin Oncol. 2002;20(3):776-790.

24. Song SP, Zhang SB, Liu R, et al. NDRG2 down-regulation and CD24 up-regulation promote tumor aggravation and poor survival in patients with gallbladder carcinoma. Med Oncol. 2012;29(3):1879-1885.

25. Wang H, Wang W, Wang X, et al. Reduced N-Myc downstreamregulated gene 2 expression is associated with $\mathrm{CD} 24$ upregulation and poor prognosis in patients with lung adenocarcinoma. Med Oncol. 2012;29(5):3162-3168.

26. Liang YX, He HC, Han ZD, et al. CD147 and VEGF expression in advanced renal cell carcinoma and their prognostic value. Cancer Invest. 2009;27(7):788-793.

27. Xuan Y, Kim S, Lin Z. Protein expression and gene promoter hypermethylation of CD99 in transitional cell carcinoma of urinary bladder. J Cancer Res Clin Oncol. 2011;137(1):49-54.

28. Ali A, Serra S, Asa SL, Chetty R. The predictive value of CK19 and CD99 in pancreatic endocrine tumors. Am J Surg Pathol. 2006;30(12): $1588-1594$.

29. Lee JH, Kim SH, Wang LH, et al. Clinical significance of CD99 downregulation in gastric adenocarcinoma. Clin Cancer Res. 2007;13(9): 2584-2591.

30. Chuang LC, Hu CY, Chen HC, et al; Community-Based Cancer Screening Program-Human Papillomavirus Study Group. Associations of human leukocyte antigen class II genotypes with human papillomavirus 18 infection and cervical intraepithelial neoplasia risk. Cancer. 2012;118(1):223-231.
OncoTargets and Therapy

\section{Publish your work in this journal}

OncoTargets and Therapy is an international, peer-reviewed, open access journal focusing on the pathological basis of all cancers, potential targets for therapy and treatment protocols employed to improve the management of cancer patients. The journal also focuses on the impact of management programs and new therapeutic agents and protocols on
Dovepress

patient perspectives such as quality of life, adherence and satisfaction. The manuscript management system is completely online and includes a very quick and fair peer-review system, which is all easy to use. Visit http://www.dovepress.com/testimonials.php to read real quotes from published authors. 